\title{
Design of complex profile extrusion dies through numerical modeling
}

\author{
N.D. Gonçalves *, O.S. Carneiro, J.M. Nóbrega \\ 1IPC/I3N - Institute for Polymer and Composites, University of Minho, Campus de Azurém, 4800-058 Guimarães, Portugal
}

\section{A R T I C L E I N F O}

\section{Article history:}

Available online 19 April 2013

\section{Keywords:}

Profile extrusion dies

Unstructured meshes

Flow balance

Computer aided design

Complex cross-section geometry

\begin{abstract}
A B S T R A C T
The achievement of a balanced flow is one of the major tasks encompassed in the design of profile extrusion dies [1]. For this purpose numerical modeling codes may be a very useful aid. The research team involved in this work has been working during the last decade on the development of numerical tools to aid the conception of extrusion dies [1,2]. The design code developed so far carries out the automatic search of a final geometry via an optimization routine coupled with geometry and mesh generators and a $3 \mathrm{D}$ computational fluid dynamics (CFD) code based on the finite volume method (FVM). This CFD code is able to model the flow of polymer melts in confined channels, but is inadequate to deal with complex geometries, since it is limited to structured meshes. This work describes the recent efforts made to enlarge the scope of the design procedures, that are currently focused on the development of a modeling code able to deal with unstructured meshes. This code solves the continuity and linear momentum conservation equations, with generalized Newtonian fluids, using a SIMPLE based approach. This paper describes the developed numerical modelling code and its employment in a case study that involves the design of a medical catheter extrusion die, focused on the search of a balanced flow distribution. The results obtained show that the developed numerical code is able to deal with complex geometrical problems, being thus a valuable tool to aid the design of extrusion dies to produce complex profiles.
\end{abstract}

(C) 2013 Elsevier B.V. All rights reserved.

\section{Introduction}

Polymer extrusion is a processing technique widely used in thermoplastic industry to produce parts for medical, building, automotive, electric and electronic applications, among others, due to its ability to generate almost any cross-section geometry profiles.

The design of a new product and corresponding extrusion die, must take into account several issues namely, achievement of balanced flow [2-4], avoidance of rheological defects, maximization of the production rate [5-7] and maintenance, or increase, of the product quality [1]. Profiles like window frames and catheters, are some of the applications that involve the most complex cross-section geometries produced by extrusion. Designing extrusion dies for the production of such geometries, requires a deep knowledge, which is usually based on experimental trial-and-error approaches, involving, therefore, the use of huge amounts of time and material resources $[8,9]$. For these reasons, the extrusion die design process can become too difficult to execute, or its cost can increase up to prohibitive levels, when complex geometry thermoplastic profiles are concerned [10,11]. To assist in the design of

\footnotetext{
* Corresponding author. Tel.: +351966955289.

E-mail addresses: nelson.goncalves@dep.uminho.pt (N.D. Gonçalves), olgasc@ dep.uminho.pt (O.S. Carneiro), mnobrega@dep.uminho.pt (J.M. Nóbrega).
}

extrusion dies, there are some commercially available softwares, e.g., POLYFLOW [12], Dieflow [13], HyperXtrude [14], FLOW 2000 [15] and PROFILECAD [16]; some of them are even able to solve the so called inverse problem [17-19], i.e., to compute the flow-channel cross-section required to produce a specific profile. Despite all the available tools, the main decisions are left to the designer [20], requiring, therefore, human intervention and knowledge. In order to automatize the extrusion die design process, some numerical codes have been developed, aiming to transfer much of those decisions to the code, e.g., the work of Ettinger [21], where the main parameters and optimization strategies required to automatize the design of profile extrusion dies are identified. However, this is restricted to 2D approaches, which limits its applicability. According to the authors knowledge, this methodology had not been further developed. Other advances on automatic optimization of profile extrusion dies have been made by Nóbrega et al. [3,5,22] using several strategies, namely those encompassing: changes performed in the flow channel parallel zone cross-section or die land, or those involving modifications performed in upstream regions. Despite of the promising results achieved, the numerical code used for this purpose is limited to simple geometry problems, inherent to the use of structured meshes. In order to extend this work to complex geometries, the use of unstructured meshes [23] is mandatory. 
The Finite Volume Method (FVM) [24] is a discretization method that can accommodate any type of grid, being, therefore, suitable to deal with complex geometries. The grid defines only the control volume (CV) boundaries and need not to be related to a coordinate system. The method is conservative by construction, as long as surface integrals (which represent convective and diffusive fluxes) are the same for the CVs sharing a common face. The FVM approach is perhaps the simplest to understand and to implement, since all terms that need to be approximated have physical meaning, fact that justifies its popularity among engineers. In addition to the discretization method, one has to select the type of grid that will discretize the problem domain. Finite Volume Method can be used with structured, block-structured or unstructured grids. The disadvantages of structured grids are its lack of adequacy for complex solution domains and the low control over the distribution of the grid points, in locations where the grid has to be refined for accuracy reasons [24]. The disadvantage of block-structured grids is that the interfaces of adjacent blocks must be treated in a fully conservative manner, which, in some cases, involve additional iterative procedures to unify the boundary conditions [24]. For very complex geometries unstructured grids are more flexible than structured grids or block-structured ones [25]. The elements, or control volumes, may have any shape and there is no restriction concerning the number of adjacent cells meeting at a point (2D) or along a line (3D). In practice, grids made of triangles or quadrilaterals in 2D, and tetrahedrons or hexahedrons in 3D, are most often used, and combinations of different type of elements can be made in the same grid. Such grids can be automatically generated and, if desired, can be orthogonal, or locally refined. The disadvantage of unstructured grids is the irregularity of data structure, that can increase the computation time required: since the matrix of algebraic equations system is irregular, the solvers are usually slower than those corresponding to regular ones. On the other hand, computer codes for unstructured grids are more flexible. They need not to be changed when the grid is locally refined, or when elements of different shapes are used. The most attractive feature of the unstructured meshes is that they allow the calculation of flows in or around geometrical features of arbitrary complexity without requiring a long time spent on mesh generation and mapping. In unstructured meshes, two different ways of defining the control volumes are possible [24-26]: in the cell-centred method the unknowns are placed at the centroid of the control volume; in the vertex-centred method they are placed on the vertices of the grid, followed by a process known as median-dual tessellation, whereby sub-volumes join centroids of the elements and midpoints of the edges. The sub-volume surrounding a node forms the control volume for discretization. Both cell-centred and vertex-centred methods are used in practice, but cell-centred method is simpler to understand and to implement, and, since a control volume always has more vertices than centroids, it has slightly lower storage requirements than the vertex-centred method.

The objective of this work is to describe a numerical code able to solve flow problems involving complex geometries, since it uses unstructured meshes, to verify it and to illustrate its use on the design of a complex extrusion die required for the production of a medical catheter. Here, the optimization of the design was done manually, i.e., it required the user intervention in the generation of the sucessive trials. However, the goal in the near future is to automatize this process $[20,21,27]$.

In the first part of this paper (Sections 2 and 3), the conservation equations and its discretization are presented. In Section 4 the implemented numerical code is verified. In Section 5 the code is used to optimize the flow balance of an extrusion die for the production of a medical catheter comprising five lumens. To reach an acceptable flow distribution at the die exit, several geometry corrections were made. Finally, in Section 6, the main conclusions of the work are drawn.

\section{Governing equations}

The Navier Stokes equations were used to model the flow with an incompressible and generalized Newtonian fluid under isothermal conditions. Taking this into account, the governing equations are the mass conservation:

$\frac{\partial u_{i}}{\partial x_{i}}=0$

and the $i$ th momentum conservation equation:

$\frac{\partial\left(\rho u_{i}\right)}{\partial t}+\frac{\partial\left(\rho u_{j} u_{i}\right)}{\partial x_{j}}=-\frac{\partial p}{\partial x_{i}}+\frac{\partial \tau_{i j}}{\partial x_{i}}$

where $u_{i}$ is the $i$ th velocity component, $\rho$ the density, $p$ the pressure and $\tau_{i j}$ is the deviatoric stress tensor, which for generalized Newtonian fluid is given by:

$\tau_{i j}=\eta(\dot{\gamma})\left(\frac{\partial u_{i}}{\partial x_{j}}+\frac{\partial u_{j}}{\partial x_{i}}\right)$

where the shear rate, $\dot{\gamma}$, is a function of the second invariant of the rate of deformation tensor:

$\dot{\gamma} \equiv \sqrt{2 \operatorname{tr} \mathbf{D}^{2}}$

$D_{i j}=\frac{1}{2}\left(\frac{\partial u_{i}}{\partial x_{j}}+\frac{\partial u_{j}}{\partial x_{i}}\right)$

A Bird-Carreau constitutive equation was employed to model the shear viscosity dependence on shear rate:

$\eta(\dot{\gamma})=\eta_{0}+\frac{\eta_{0}-\eta_{\infty}}{\left(1+(\lambda \dot{\gamma})^{2}\right)^{\frac{1-n}{2}}}$

being $\eta_{0}$ the zero shear-rate viscosity, $\eta_{\infty}$ the viscosity at very high shear rates, $\lambda$ a characteristic time and $n$ the power-law index.

Due to the explicit nature of the constitutive equation (Eq. (3)) it can be directly inserted in the momentum conservation equations, giving:

$\frac{\partial\left(\rho u_{i}\right)}{\partial t}+\frac{\partial\left(\rho u_{j} u_{i}\right)}{\partial x_{j}}-\frac{\partial}{\partial x_{j}}\left(\eta(\dot{\gamma}) \frac{\partial u_{i}}{\partial x_{j}}\right)=-\frac{\partial p}{\partial x_{i}}$

which comprises (from left to right) the following terms: unsteady, advective, diffusive and pressure source.

\section{The numerical calculation procedure}

The governing equations were discretized following the FVM for unstructured meshes, which starts by integration on each control volume $(\Omega)$ and time step $(\Delta t)$. In this way, the integral form of the equation can be written as a sum of integrals of each term, which is described hereafter.

For the momentum conservation equation (Eq. (7)) the unsteady term is considered constant inside the control volume, and its integration over the time interval is discretized using a first order (backward) differencing scheme [25]. This simple approach was employed since the problems solved are steady and time evolution is used just for relaxation purposes.

To avoid the decoupling between pressure and velocity fields, the normal component of velocity at the cell faces, required for the advective term, is computed using the Rhie and Chow [25] interpolation scheme, whereas scalar velocity is computed using a Total Variation Diminishing (TVD) interpolation scheme [25]. 
The gradients required for the advective, diffusive and pressure source terms were computed using the least square method and the values from the previous time step ([25]).

For the momentum equation four boundary conditions were considered:

- inlet, with imposed velocity $u_{i}=u_{i, \text { inlet }}$

- outlet, null normal gradients $\frac{\partial u_{i}}{\partial x_{i}} n_{i}=0$

- wall, with null velocity $u_{i, \text { wall }}=0_{i}$ and

- symmetry, $u_{i, \text { wall }} n_{i, \text { wall }}=0$.

As mentioned before, to couple velocity and pressure fields, the normal velocities of the inner faces are computed using a Rhie and Chow [25] based interpolation scheme:

$$
\begin{aligned}
u_{j . f} n_{j, f}= & \frac{u_{P . f}+u_{n b . f}}{2} n_{j . f}+\frac{1}{2}\left(\frac{\Delta V_{P}}{a_{P}}+\frac{\Delta V_{n b}}{a_{n b}}\right) \frac{p_{P}-p_{n b}}{\|\xi\|} \\
& -\frac{1}{2}\left(\frac{\Delta V_{P}}{a_{P}}\left(\frac{\overline{\partial p}}{\partial x_{j}}\right)_{P}+\frac{\Delta V_{n b}}{a_{n b}}\left(\frac{\overline{\partial p}}{\partial x_{j}}\right)_{n b}\right) \frac{\xi_{j . f}}{\|\xi\|}
\end{aligned}
$$

where the gradient $\frac{\partial p}{\partial x_{j}}$ values are estimated with least squares method, $\Delta V_{P}$ is the $\mathrm{P}$ cell volume, $\Delta V_{n b}$ is the neighbor's cell volume, $a_{P}$ and $a_{n b}$ are the diagonal coefficients of cells $P$ and $n b$ of the momentum conservation equation [25].

The mass conservation equation (Eq. (1)) is used to obtain a pressure correction equation for each control volume. For this purpose, the equation is integrated on the control volume, then, the Gauss' Divergence Theorem is applied and the velocity is written as a sum of an estimated $\left(\mathbf{u}^{*}\right)$ and correction $\left(\mathbf{u}^{\prime}\right)$ velocities:

$\sum_{f} A_{f}\left(u_{j, f}^{*} n_{j, f}+u_{j, f}^{\prime} n_{j, f}\right)=0$

Computing the difference between the correct face velocity values, obtained with Eq. (8), and those obtained with a similar one to compute the face estimated values $u_{j, f}^{*} n_{j, f}$, an expression for the normal velocity face correction is obtained:

$u_{j . f}^{\prime} n_{j, f}=\frac{1}{2}\left(\frac{\Delta V_{P}}{a_{P}}+\frac{\Delta V_{n b}}{a_{n b}}\right) \frac{p_{P}^{\prime}-p_{n b}^{\prime}}{\|\xi\|}$

Substituting Eq. (10) in Eq. (9) a equation for the pressure field correction is obtained, for each cell:

$\sum_{f} \frac{A_{f}}{2\|\xi\|}\left(\frac{\Delta V_{P}}{a_{P}}+\frac{\Delta V_{n b}}{a_{n b}}\right)\left(p_{P}^{\prime}-p_{n b}^{\prime}\right)=-\sum_{f} A_{f} u_{j . f}^{*} \cdot n_{j, f}$

where the right hand side corresponds to the mass imbalance of each cell.

The pressure field correction, together with Eq. (10), allow to correct the normal face velocities. For the cell velocities a correction expression can be obtained by subtracting the momentum conservation equations for the estimated and correct fields, which gives:

$\sum_{n b} a_{n b} u_{i, n b}^{\prime}+a_{P} u_{i, P}^{\prime}=-\frac{\partial p^{\prime}}{\partial x_{i}} \Delta V_{P}$

As in the main approach of SIMPLE [25], if the neighbors contributions are neglected, an explicit expression to cell velocity correction is obtained

$u_{i, P}^{\prime}=-\frac{\Delta V_{P}}{a_{P}} \frac{\partial p^{\prime}}{\partial x_{i}}$

where the pressure correction gradient is computed using a least squares method [25].

The full calculation procedure is illustrated in the flowchart depicted in Fig. 1.

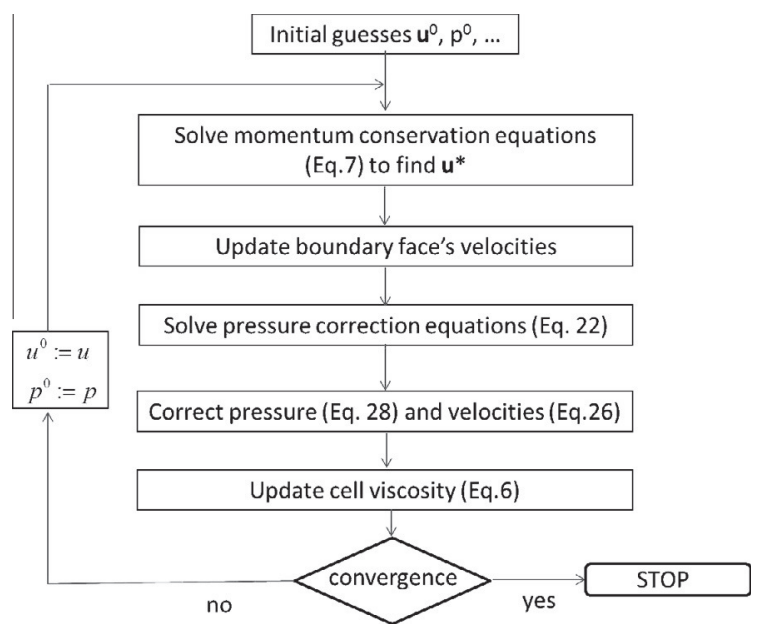

Fig. 1. Code flowchart.

The process starts with estimated values for velocity and pressure $\left(u^{0}\right.$ and $\left.p^{0}\right)$. Then, the momentum conservation equations are solved. Subsequently, the boundary velocities at the outlet are computed using the appropriate boundary condition, and then corrected in order to equalize the outlet flow to the inlet flow. After updating the velocities on boundaries, the pressure correction system is built and solved, enabling to perform the correction of pressures and velocities. To avoid divergence, the pressures on the cells are corrected with a relaxation factor [25] $\alpha$ (it is common to use a value of 0.1 ), through the following equation: (see Fig. 2)

$p=p^{*}+\alpha p^{\prime}$

The process restarts if the error residuals are larger than a predefined value.

\section{Code verification}

To verify the code, three typical benchmark problems were tested: the Poiseuille flow between parallel plates, which has an analytical solution, and the Lid-driven Cavity and flow around a cylinder problems, whose numerical solutions are given by Ghia et al. [28] and Bharti et al. [29], respectively.

\subsection{Poiseuille flow between parallel plates}

This problem was solved considering a Generalized Newtonian fluid that follows a Power-law constitutive equation with $n=1$ (particular case of a Newtonian fluid) and $n=0.7$. For the mesh sensitivity studies, four meshes were employed with 5, 10, 20 and 40 cells along the channel thickness, which were designated by M0, M1, M2 and M3, respectively.

The numerical values predicted for the axial pressure gradient, $p_{x}$, and maximum channel velocity $\left(u_{\max }\right)$ were compared with their analytical counterparts given by Eqs. (15) and (16). The results obtained, plotted in Fig. 3, allow to confirm that the predicted numerical values tend to the analytical ones as the mesh is refined.

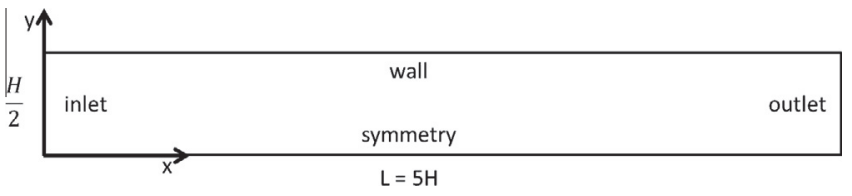

Fig. 2. Poiseuille problem - geometry and boundary conditions. 


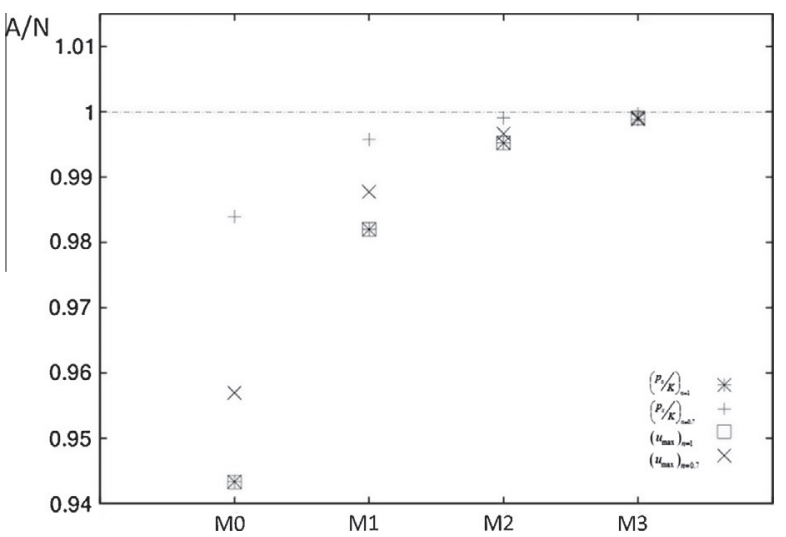

Fig. 3. Mesh sensitivity study for the ratio between analytical (A) and numerically predicted $(\mathrm{N})$ values, for maximum velocity $u_{\max }$ and axial pressure gradient $\left(p_{x} / K\right)$, for the Poiseuille case study with Newtonian $(n=1.0)$ and Generalized Newtonain $(n=0.7)$ fluids.

It is also worth to notice that with M1 the errors obtained are below $2 \%$.

$\frac{p_{x}}{K}=\frac{-\left(\frac{1+2 n}{n H} U\right)^{n}}{H}$

$u(y)=\left(\frac{-p_{x}}{K}\right)^{\frac{1}{n}} \frac{H^{1+\frac{1}{n}}}{1+\frac{1}{n}}\left(1-\left(\frac{y}{H}\right)^{1+\frac{1}{n}}\right)$

For assessment purposes, the velocity profiles obtained for the two cases considered ( $n=1 ; K=1 \mathrm{~Pa} \mathrm{~s}$ and $n=0.7 ; K=1 \mathrm{~Pa} \mathrm{~s}{ }^{0.7}$ ), with mesh M3, are plotted in Fig. 4.

These results allow to confirm the code accuracy when dealing with generalized Newtonian Fluids.

\subsection{Lid-driven cavity}

The Lid-driven cavity is another benchmark problem usually employed to verify numerical codes. This problem consists of a square channel with three fixed walls and a tangential homogeneous velocity boundary on the top, as shown in Fig. 5a. The flow velocity was defined in order to have a Reynolds number $(R e)$ of 100.

A mesh with $129 \times 129$ cells was used, as illustrated in Fig. 5b, with smaller cells close to the walls, where higher velocity gradients are expected.

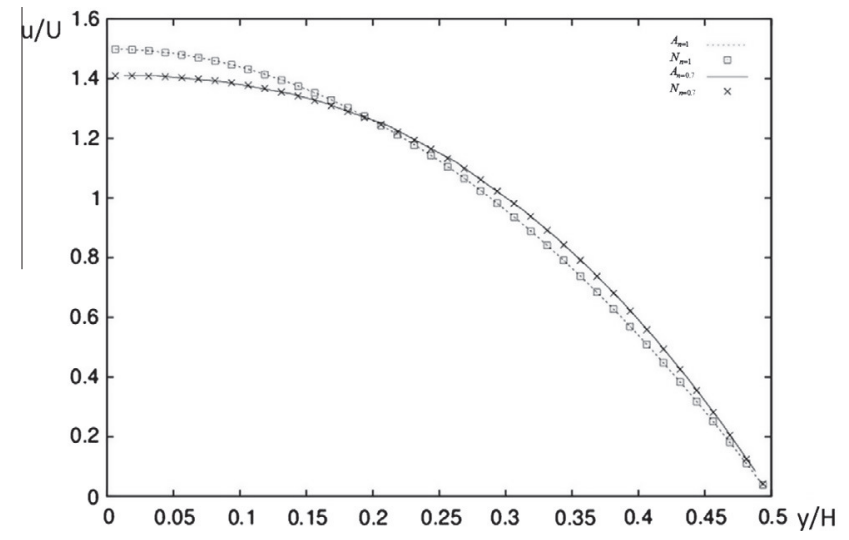

Fig. 4. Comparison between the analytical (A) and numerically predicteed $(\mathrm{N})$ normalized velocity profiles, for the Poiseuille case study with Newtonian $(n=1.0)$ and Generalized Newtonian ( $n=0.7$ ) fluids.

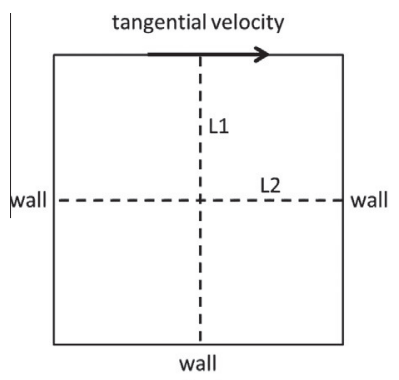

(a)

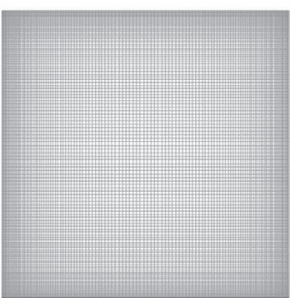

(b)
Fig. 5. Lid-driven cavity: (a) boundary conditions; (b) mesh with $129 \times 129$ cells.

The results obtained with the developed numerical code and those obtained by Ghia et al. [28] for the $u$ and $v$ velocity components along $L 1$ and $L 2$ lines (see Fig. 5a), are shown in Fig. 6. Both velocity components computed with the new code, evidenced a good correlation with those of Ghia et al. [28].

The streamlines predicted by the developed numerical code and by Ghia et al. are presented in Fig. 7a and b, respectively. The similarity of both results, in terms of number, position and size of the vortices, comprises an additional validation for the implementation.

\subsection{Flow around a cylinder}

The flow around a cylinder, one of the most extensively studied problems in fluid mechanics $[29,30]$, was used in this work to

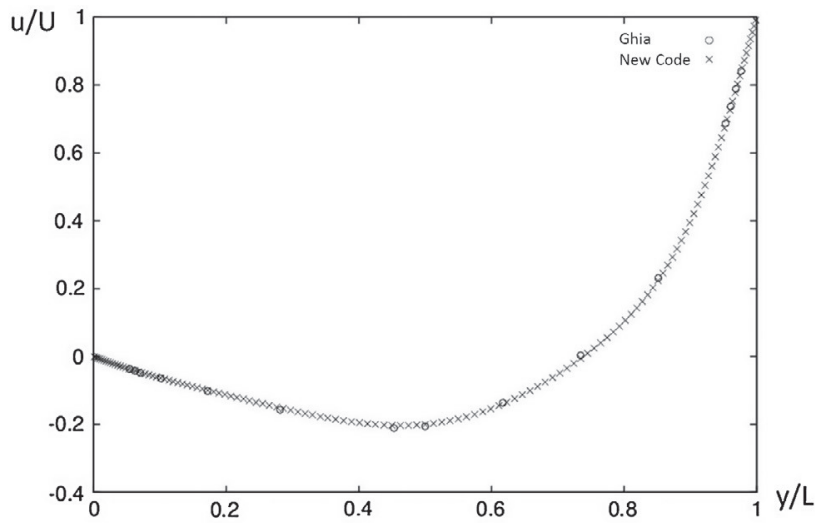

(a)

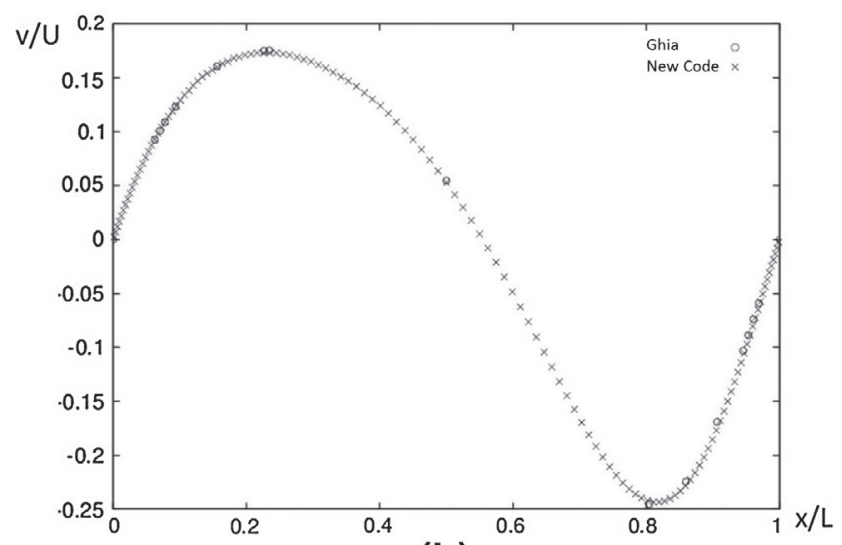

(b)

Fig. 6. Velocity for the Lid-driven cavity case study: (a) $u$ velocity along $L 1$ (Fig. 5a); (b) $v$ velocity along $L 2$ (Fig. 5 a) 


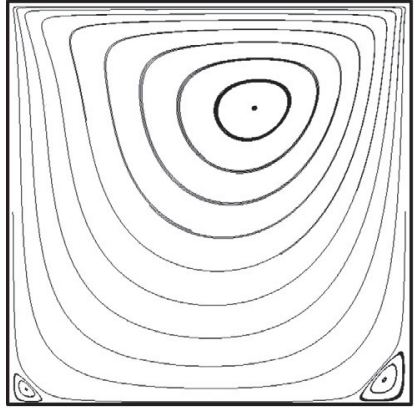

(a)

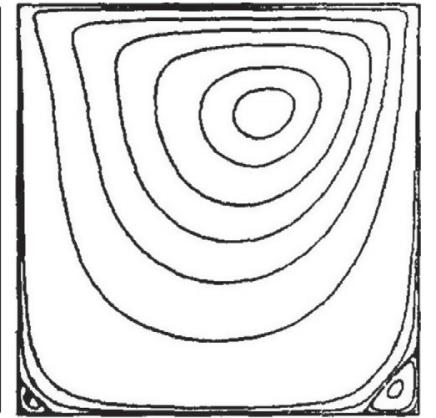

(b)
Fig. 7. Lid-driven cavity problem - streamlines: (a) new code; (b) Ghia et al. [28]

verify the accuracy of the numerical predictions on a complex geometry.

The geometry used was, as suggested by Bharti et al. [29], a square with edge lengths $2 L$, having a circle of diameter $D$ in its center, as illustrated in Fig. 8. As shown in this figure, due to symmetry reasons just half of the geometry was considered for the numerical model, and adequate boundary conditions were employed. The other boundary conditions set, were a constant velocity of $1 \mathrm{~m} \mathrm{~s}^{-1}$ (corresponding to $R e=10$ ) for the inlet, null gradients at the outlet and a no slip wall condition, $\mathbf{u}_{\text {wall }}=\mathbf{0}$, on the cylinder wall.

As suggested by Bharti [29], a geometry with $L=30.5 D$ was used, since it was found adequate to produce almost domain independent results.

For the numerical models, the coarsest mesh employed (M0) has 75 cells along each symmetry line, 10 cells allong each vertical boundary, 20 cells along top boundary (Fig. 9a) and 100 cells along the cylinder edge (Fig. 9b). On the bottom symmetry lines a progression factor was used.

The meshes M1, M2 and M3 were obtained based on M0, successively doubling the number of cells along each boundary. The number of cells in each mesh is 5148, 21,488, 88,854 and 355,298 , respectively, for M0-M3. Here it is important to notice that for the benchmark problem considered, Bharti et al. [29], used 101 cells along the cylinder edge, for the finest mesh.

To compare the numerical code predictions with similar results obtained by Bharti et al. [29], two parameters, illustrated in Fig. 8, were calculated: the length of wake (or recirculation) zone $\left(L_{w}\right)$, that is the dimensionless distance measured from the rear of the cylinder to the point of the attachment for the near closed streamline on the downstream symmetry edge, and the angle of separation $\left(\theta_{S}\right)$, that is measured from the rear stagnation point to the onset of the flow separation from the solid surface.

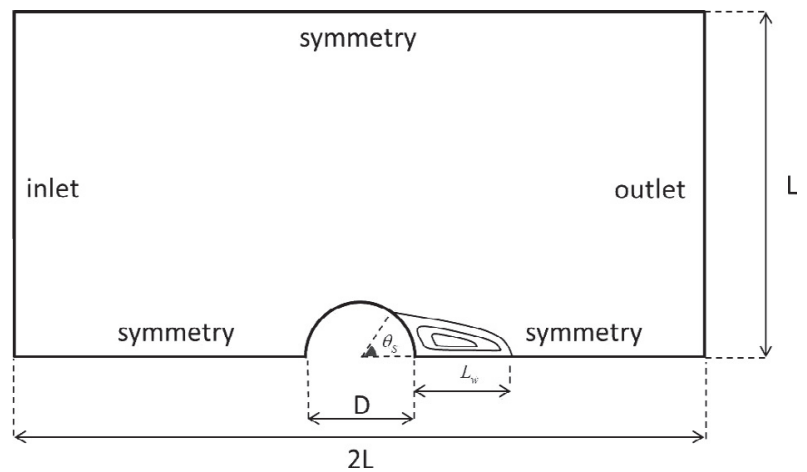

Fig. 8. Flow around a cylinder problem - geometry and boundary conditions

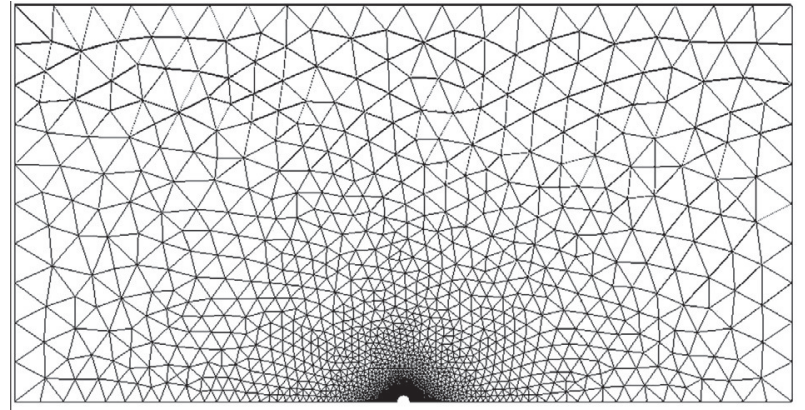

(a)

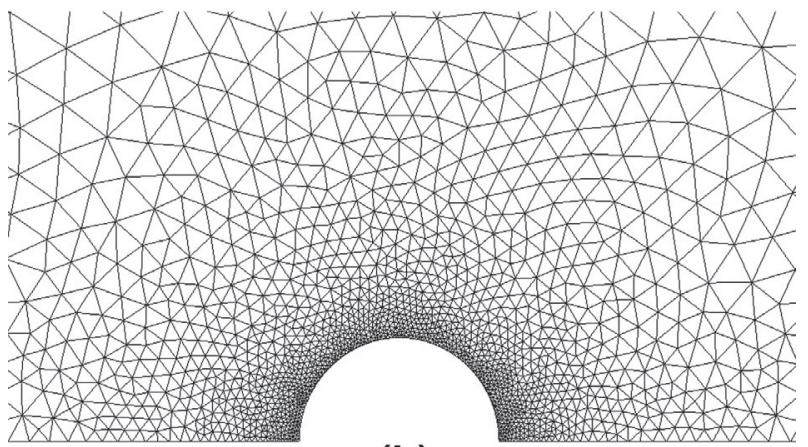

(b)

Fig. 9. Flow around a cylinder problem - mesh: (a) full mesh; (b) close-up view around the cylinder.

The results plotted in Fig. 10 allow to verify that the numerically predicted and benchmark results computed tend to slightly different values. For the finest meshes used in both works circa $3.2 \%$ and $1.7 \%$ difference was obtained, respectively, for the length of wake $\left(L_{w}\right)$ and angle of separation $\left(\theta_{S}\right)$. However, as mentioned before, the meshes employed in this work were much finer than the ones employed by Barthi et al. [29], e.g., circa 8 times more cells along cylinder edge were used in our meshes. Regarding the number of cells along the cylinder edge, the meshes employed by Barthi et al. [29] are equivalent to M0. Additionaly, Barthi et al. refer [29] that their results were obtained with a accuracy of circa $1-2 \%[29]$.

This case study allowed to conclude that the developed numerical code allows to perform accurate computations on complex geometries.

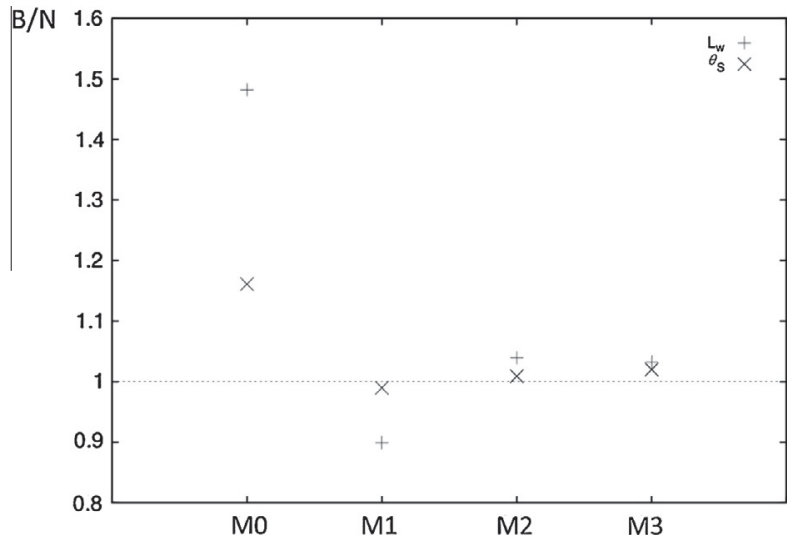

Fig. 10. Mesh sensitivity study for the ratio between benchmark (B) and numerically predicted $(\mathrm{N})$ values, for the length of wake $\left(L_{w}\right)$ and angle of separation $\left(\theta_{S}\right)$, for the flow around a cylinder case study. 


\section{Design of a catheter extrusion die}

The developed code was used to optimize the flow distribution in an extrusion die designed for the production of a medical catheter profile, that comprises a complex geometry cross-section with five internal channels (lumens), shown in Fig. 11a.

The material employed was a polypropylene homopolymer extrusion grade, Novolen PPH 2150, from Targor. Its rheological behaviour was experimentally characterised in capillary and rotational rheometers, at $230^{\circ} \mathrm{C}$ [3]. The shear viscosity data were fitted with the least-squares method by means of the Bird-Carreau constitutive equation (Eq. (6)), considering $\eta_{\infty}=0$ Pa s, yielding the following parameters: $\eta_{0}=5.58 \times 10^{4} \mathrm{~Pa} \mathrm{~s}, \lambda=3.21 \mathrm{~s}$ and $n=0.3014$.

The inlet velocity was set in order to have a outlet average velocity of circa $1.2 \mathrm{~m} \mathrm{~min}^{-1}$.

The full flow channel geometry comprises the region depicted in Fig. 11b and an additional convergent circular region that

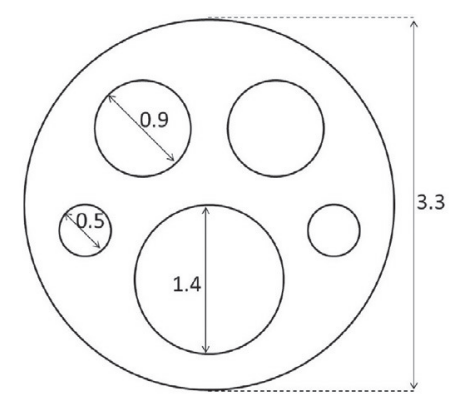

(a)

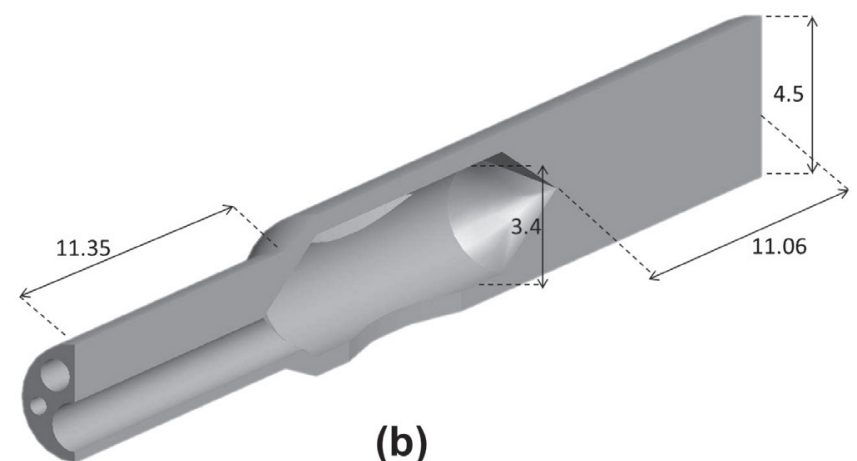

Fig. 11. Medical catheter: (a) profile cross-section; (b) flow channel geometry (dimensions in $\mathrm{mm}$ ).

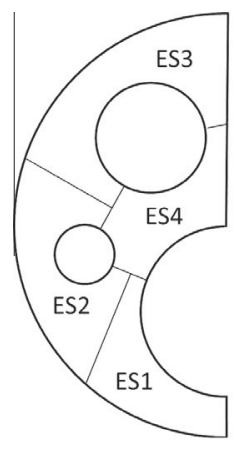

(a)

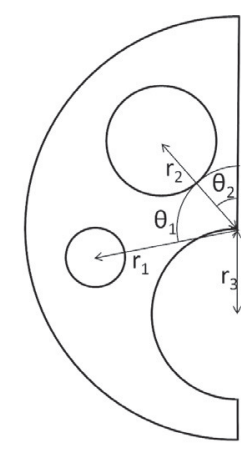

(b)
Fig. 12. Catheter cross-section: (a) elemental sections; (b) controllable parameters.

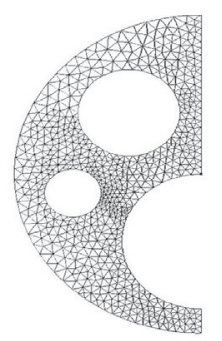

(a)

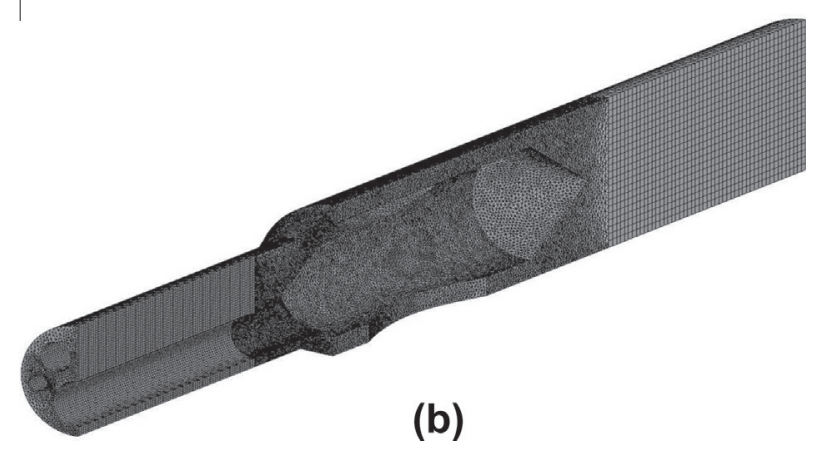

Fig. 13. Catheter mesh: (a) outlet face mesh; (b) global domain mesh with circa 390,000 cells.

connects this region to the extruder. However, since this convergent region will not influence the flow distribution, the flow was modeled just in the geometry shown in Fig. 11b.

Due to the different restrictions promoted to the flow along the channel cross-section, which is a direct consequence of the differential thicknesses comprised by the profile, the flow will be naturally unbalanced, resulting in different average linear velocities at the flow channel outlet, which is commonly referred as an unbalanced flow distribution [2]. Since it is not possible to produce the required geometry when the flow is not properly balanced, one has to find a strategy to obtain a more even flow distribution.

To analise the flow distribution at the outlet section, the geometry is divided into 4 Elemental Sections (ES) [31], as shown in Fig. 12a, where the average velocity is computed.

The quality of the flow distribution was evaluated by an objective function that is always positive and becomes zero when all the ESs reaches the bulk average velocity, that is given by

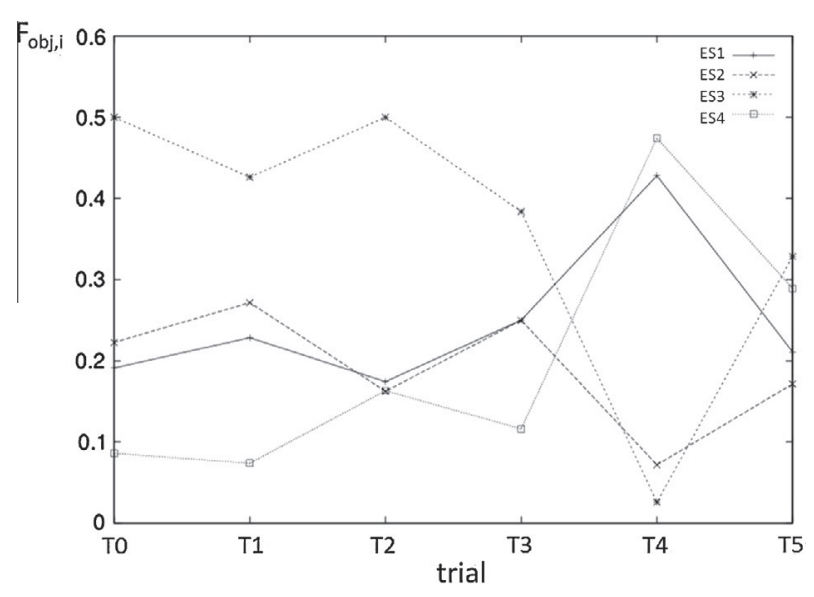

Fig. 14. contribution of each ES for the objective function. 


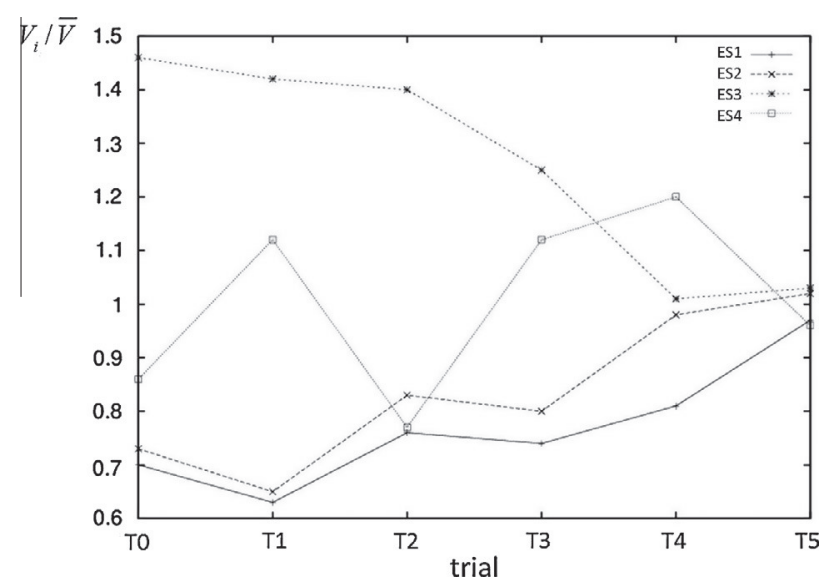

Fig. 15. outlet average velocity (normalized by outlet global average velocity).

$F_{o b j}=\sum_{i} F_{o b j, i}=\sum_{i}\left\|1-\frac{\bar{V}}{\bar{V}}\right\| \frac{A_{i}}{A}$

where $\bar{V}_{i}$ is the average flow velocity at ES $i, \bar{V}$ is the global outlet average flow velocity, $A_{i}$ is the area of ES $i$ and $A$ is the total outlet area.

The contribution of each ES to the objective function can be quantified by $F_{o b j, i}$ that gives information on the local flow unbalance. These values were employed to guide the flow channel geometry corrections. Thus, for each trial, the ES that had a greater contribution to the objective function was identified, and the

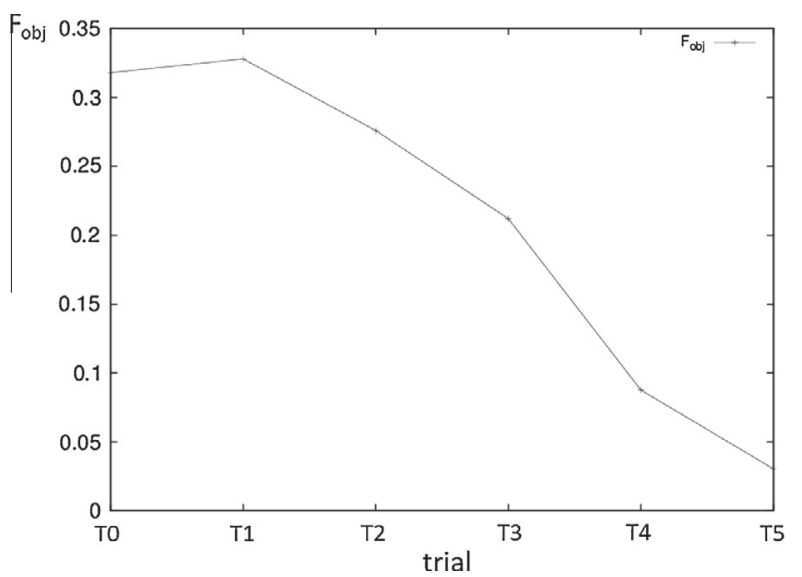

Fig. 17. objective function evolution in consecutive trials.

appropriate controllable geometric parameters(s) that affect its velocity was (were) changed, with a view to improve its performance.

The relevant dimensions of the cross-section of the catheter are shown in Fig. 11a. In order to be able to balance the flow distribution at the channel outlet, some geometric parameters can be changed to control the flow distribution. These are the angles and distance to the cross-section centre, one for each hole (lumen), as illustrated in Fig. 12b. The changes in these parameters are not expected to affect the catheter performance, since the channels (lumens) functionality is maintained when their cross area is kept

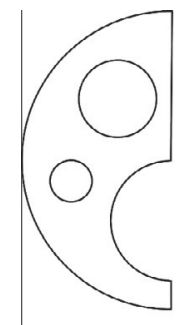

TO

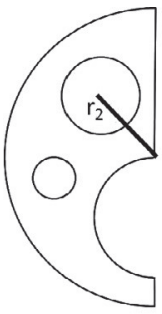

T 1

$r_{2}$

from 0.90

to 0.95

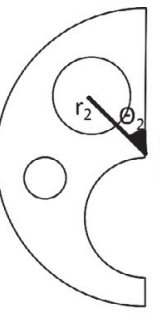

T 2

$r_{2}$

from 0.95

to 0.90

$\Theta_{2}$

from $45^{\circ}$

to $43^{\circ}$

(a)

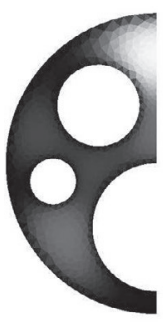

T 0

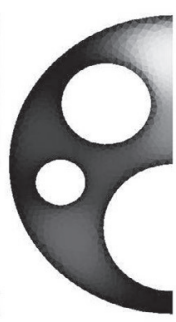

T 1

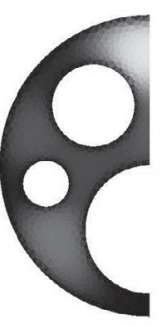

T 2
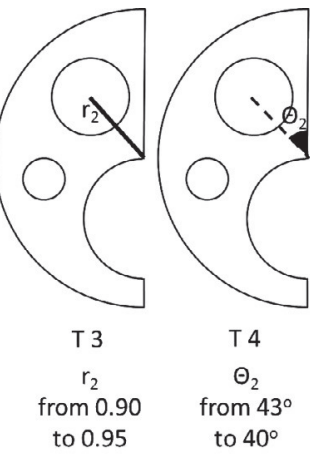

T 4

$\Theta_{2}$

from $43^{\circ}$

to $40^{\circ}$

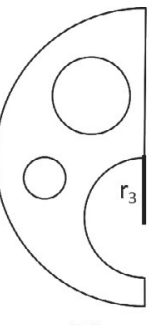

T 5

from 0.7

to 0.65

normalized velocity

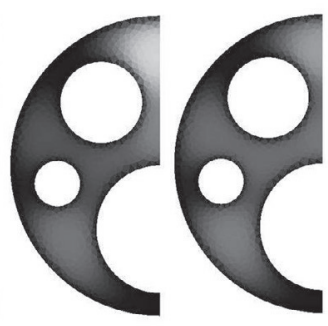

T 4

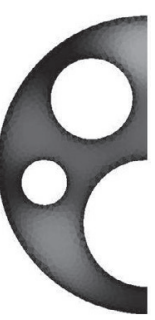

T 5

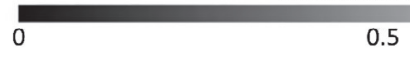

(b)

Fig. 16. catheter - outlet: (a) geometry changes; (b) outlet velocity field. 
unchanged. The initial values of these parameters were $r_{1}=1.15 \mathrm{~mm}, \theta_{1}=45^{\circ}, r_{2}=0.9 \mathrm{~mm}, \theta_{2}=100^{\circ}$ and $r_{3}=0.7 \mathrm{~mm}$.

To compute the flow field a mesh with circa 390,000 cells was used, comprising triangular prisms (circa 166,000) in constant cross sector zones and tetrahedrons (circa 224,000) elsewhere. The typical meshes used on the outlet face and on the global domain, can be seen in Fig. 13a and in Fig. 13b, respectively.

The contribution of each ES for the objective function is presented in Fig. 14, and the evolution of the average velocity in each $\mathrm{ES}$, normalized by the outlet global average velocity, is presented in Fig. 15, where it can be seen that after five trials a similar average velocity was attained in all ES. The methodology used to improve the performance of the die in subsequent trials was previously described. According to it, and as an example, in Fig. 14, one can see that on trial T0, ES3 is the one that contributes more to the global objective function, and in Fig. 15, it can be seen that it has the highest average flow velocity. To correct this problem, on trial T1 a change on the controllable parameters was done (Fig. 16a), in order to increase the restriction of the flow in this ES (ES3). There were several possibilities to achieve this objective, including a change on $r_{2}$ (trial T1), $\theta_{2}$ (trial T2) or a combination of both (trial T3). As one can see in Fig. 17, the change on $\theta_{2}$ led to better results than the change on $r_{2}$, and the combination of changes in both parameters resulted even in a better improvement on performance. Having this in mind, and because the ES with higher contribution to objective function remained ES3, on trial T4 $\theta_{2}$ was changed again. Finally, as the main contribution to the objective function changed to ES4, and its average velocity is higher than the other ES average velocities, $r_{3}$ was decreased on trial T5 in order to restrict the flow in this ES.

As one can see in Fig. 17, after five trials the objective function decreased circa one order of magnitude. The improvements obtained for the velocity field are depicted in Fig. 16b, evidencing that the final flow distribution is significantly better than the initial one.

\section{Conclusions}

In this work a numerical code able to deal with complex geometries and unstructured meshes, developed to predict the flow field of polymer melts in confined channels, using generalized Newtonian flow models, was described and verified. The developed code was then used to improve the flow distribution in a complex profile extrusion die, for the production of a medical catheter. The results obtained show that the developed numerical code is a very useful tool to aid the design of extrusion dies used in the production of complex geometry profiles.

\section{Acknowledgements}

The authors gratefully acknowledge funding from Fundação para a Ciência e Tecnologia through the PhD Grant SFRH/ BD43632/2008 and FCT (COMPETE Program) under the Projects FCOMP-01-0124 - FEDER-010190 (Ref. PTDC/EME - MFE/ 102729/2008) and FCOMP-01-0124-FEDER-015126 (Ref ${ }^{a}$. FCT PTDC/EME-MFE/113988/2009), and FEDER, via FCT, under the PEst-C/CTM/LA0025/2011 (Strategic Project - LA 25 - 2011-2012).

\section{References}

[1] V. Hristov, J. Vlachopoulos, Thermoplastic silicone elastomer lubricant in extrusion of polypropylene wood flour composites, Advances in Polymer Technology 26 (2007) 100-108.

[2] J.M. Nóbrega, O.S. Carneiro, F.T. Pinho, P.J. Oliveira, Flow balancing in extrusion dies for thermoplastic profiles - part iii: Experimental assessment, International Polymer Processing 19 (2004) 225-235.

[3] J.M. Nóbrega, O.S. Carneiro, F.T. Pinho, P.J. Oliveira, Flow balancing in extrusion dies for thermoplastic profiles - part i: Auromatic design, International Polymer Processing 18 (3) (2003) 298-306.

[4] J.M. Nóbrega, O.S. Carneiro, P.J. Oliveira, F.T. Pinho, Flow balancing in extrusion dies for thermoplastic profiles: non-isothermal effects, in: 17th Annual Conference of the Polymer Processing Society, PPS-17, 2001, pp. 1-12.

[5] O.S. Carneiro, J.M. Nóbrega, F.T. Pinho, P.J. Oliveira, Computer aided rheological design of extrusion dies for profiles, Journal of Materials Processing Technology 114 (2001) 75-86.

[6] J.M. Nóbrega, O.S. Carneiro, A. Gaspar-Cunha, N.D. Gonçalves, Design of calibrators for profile extrusion - optimizing multi-step systems, International Polymer Processing 23 (3) (2008) 331-338.

[7] J.M. Nóbrega, O.S. Carneiro, J. a Covas, F.T. Pinho, P.J. Oliveira, Design of calibrators for extruded profiles. Part i: Modeling the thermal interchanges, Polymer Engineering and Science 44 (12) (2004) 2216-2228.

[8] R.J. Brown, H.T. Kim, J.W. Summers, Pratical principles of die design, in: SPE, 37th Annual Technical Conference, 1979.

[9] J.H. Schut, Computer flow analysis helps develop new profile dies faster, Plastic Technology, 2003.

[10] W. Michaeli, Extrusion Dies for Plastic and Rubber: Design and Engineering Computations, second ed., Hanser Publishers, 1992.

[11] Z. Tadmor, c. Cogos, Principles of Polymer Processing, second ed., John Wiley and Sons Inc., 2006.

[12] Ansys polyflow, <http://www.ansys.com/Products/Simulation+Technology/ Fluid+Dynamics/ANSYS+Polyflow $>$

[13] Dieflow, <http://www.dieflow.com/>.

[14] Hyperxtrude, <http://www.altairhyperworks.com/Product,15,HyperXtrude. aspx>.

[15] Flow 2000, <http://www.futuresoft.net/F2k-ext.htm>.

[16] Profilecad, <http://www.polydynamics.com/profile.htm>.

[17] V. Legat, J.-M. Marchal, Three-dimensional extrusion: an implicit formulation for gn flows, in: Theorectical and Applied Rheology, Proc. XIth Int. Congr. on Rheology, Elsevier Science Publishers B.V., 1992.

[18] V. Legat, J.M. Marchal, Die design - an implicit formulation for the inverse problem, International Journal for Numerical Methods in Fluids 16 (1) (1993) 29-42.

[19] J.M. Marchal, A. Goublomme, Parametric optimization of extrusion dies through numerical simulation, in: 3rd Esaform Conference on Material Forming, 2000.

[20] A.R. Shahreza, A.H. Behravesh, M.B. Jooybari, E. Soury, Design, optimization, and manufacturing of a multiple-thickness profile extrusion die with a cross flow, Polymer Engineering and Science 50 (2010) 2417-2424.

[21] H.J. Ettinger, J. Sienz, J.F.T. Pittman, A. Polynkin, Parameterization and optimization strategies for the automated design of upvc profile extrusion dies, Structural and Multidisciplinary Optimization 28 (2004) 180-194.

[22] O.S. Carneiro, J.M. Nóbrega, P.J. Oliveira, F.T. Pinho, Flow balancing in extrusion dies for thermoplastic profiles. Part ii: Influence of the design strategy, International Polymer Processing 18 (3) (2003) 307.

[23] N.D. Gonçalves, Finite volume method on unstructured meshes (in portuguese), Master's thesis, Faculdade de Ciências da Universidade do Porto, 2007.

[24] J.H. Ferziger, M. Peric, Computatinal Methods for Fluid Dynamics, third ed., Springer, 2002.

[25] H.K. Versteeg, W. Malalasekera, An Introduction to Computational Fluid Dynamics - The Finite Volume Method, second ed., Pearson, 2007.

[26] J. Blazek, Computational Fluid Dynamics: Principles and Applications, second ed., Elsevier Science, 2006.

[27] J. Sienz, A. Goublomme, M. Luege, Sensitivity analysis for the design of profile extrusion dies, Computers \& Structures 88 (9-10) (2010) 610-624.

[28] U. Ghia, K.N. Ghia, C.T. Shin, High-re solutions for incompressible flow using the Navier-Stokes equations and a multigrid method, Journal of Computational Physics 48 (1982) 387-411.

[29] R.P. Bharti, R.P. Chhabra, V. Eswaran, Steady flow of power law fluids across a circular cylinder, The Canadian Journal of Chemical Engineering 84 (2006) 406-421.

[30] S.K. Panda, R.P. Chhabra, Laminar flow of power-law fluids past a rotating cylinder, Journal of Non-Newtonian Fluid Mechanics 165 (2010) 14421461.

[31] O.S. Carneiro, J.M. Nóbrega, F.T. Pinho, P.J. Oliveira, Automatic balancing of profile extrusion dies: experimental assessment, in: ANTEC 2004, SPE Annual Technical Conference, vol. 50, 2004, pp. 91-95. 